esting problem in tumor biology, as instability is one of the main reasons for current therapeutic failures and acquired resistance in cancer therapy. Thus, molecular targeting of pathways responsible for genomic instability or selective killing of cells carrying chromosomal imbalances would have a tremendous impact in the clinical management of individuals with cancer.

1. DePinho, R.A. Nature 408, 248-254 (2000).

2. Storchova, Z. \& Pellman, D. Nat. Rev. Mol. Cell. Biol. 5, 45-54 (2004).

3. Artandi, S.E. et al. Nature 406, 641-645 (2000).

4. Romanov, S.R. et al. Nature 409, 633-637 (2001).

5. Rudolph, K.L. et al. Nat. Genet. 28, 155-159
(2001)

6. van Heek, N.T. et al. Am. J. Pathol. 161, 1541-1547 (2002).

7. Chin, K. et al. Nat. Genet. 36, 984-988 (2004)

8. Ma, X.J. et al. Proc. Natl. Acad. Sci. USA 100, 5974-5979 (2003).

9. Porter, D. et al. Mol. Cancer Res. 1, 362-375 (2003).

10. Boecker, W. et al. J. Pathol. 195, 415-421 (2001).

11. Chin, L. et al. Cell 97, 527-538 (1999).

\title{
TIMP3 checks inflammation
}

\author{
Roy A Black
}

Mice deficient in the metalloprotease inhibitor TIMP3, which inhibits the tumor-necrosis factor alpha (TNF- $\alpha$ )converting enzyme (TACE, also called ADAM17), have elevated levels of TNF and severe inflammation in the liver. This result confirms the physiological importance of the soluble form of TNF and identifies TIMP3 as a crucial regulator of this inflammatory cytokine.

TIMP3 was first identified, inauspiciously, as a chicken protein similar to two wellstudied mammalian inhibitors of matrixdegrading metalloproteases ${ }^{1}$, but its biological importance has become increasingly apparent. It induces apoptosis ${ }^{2}$, inhibits angiogenesis ${ }^{3}$ and impedes cell migration $^{4}$, at least in tissue culture or when overexpressed in vivo. Whether these effects occur physiologically is not known. In a new study on page 969, Fazilat Mohammed and colleagues ${ }^{5}$ show that TIMP3 is a physiological regulator of inflammation. The authors found that mice lacking TIMP3 develop inflamed livers, and that the cause of this inflammation is an increase in TNF- $\alpha$ activity.

All four known TIMPs ${ }^{6,7}$ control the matrix-degrading metalloproteases, which have roles in many normal and pathological processes involving extracellular matrix turnover and cell migration ${ }^{8}$. TIMP3, uniquely, also inhibits TACE, a protease that generates soluble TNF from the cellsurface form of the cytokine $\mathrm{e}^{9-11}$. Consistent with this in vitro activity, TIMP3 blocks the release of TNF from cells in tissue culture ${ }^{12}$. TNF is a key inflammatory mediator that causes severe liver damage by a variety of mechanisms ${ }^{13}$ (Fig. 1). The demonstration by Mohammed et al. ${ }^{5}$ that TIMP3 controls TNF levels in vivo, and that this check prevents spontaneous inflammation, is a landmark in the TIMP, TNF and inflammation fields.

Roy A. Black is at Amgen, 1201 Amgen Court W., Seattle, Washington, USA.

e-mail:blackra@amgen.com

\section{The TIMP3 cascade}

Mohammed et al. readily detected TNF in liver homogenates from Timp $3^{-1-}$ mice, but not in those from wild-type mice or in any other tissues from Timp $3^{-/-}$mice that they tested. TACE activity was greater in liver homogenates from Timp $3^{-1-}$ mice than in those from wildtype mice. They observed predicted consequences of TNF signaling, such as NFKB activation and IL- 6 production, in livers of Timp $3^{-/-}$mice. By 22 months of age, livers of these mice showed lymphocytic infiltrates and necrosis. Timp $3^{-1-}$ mice that also lacked the type I TNF receptor (the primary transducer of the inflammatory effects of TNF) showed none of these signs of inflammation. This finding confirmed that the inflammatory damage was due to TNF activity. Livers of Timp $3^{-1-}$ mice that had undergone a partial hepatectomy had even higher levels of TNF, which persisted well beyond the transient rise seen in livers of wildtype mice that had undergone a partial hepatectomy. Livers of Timp $3^{-1-}$ mice showed severe necrosis after initially normal regeneration,

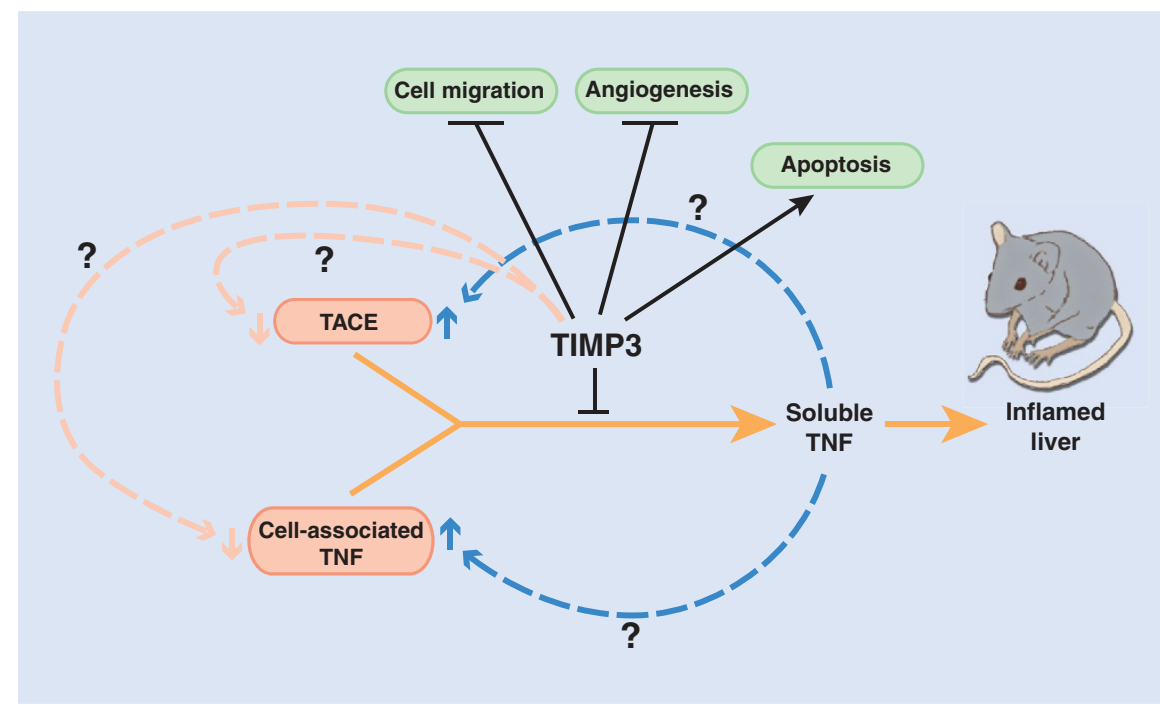

Figure 1 The simplest explanation for the results reported by Mohammed et al. ${ }^{5}$ is that TIMP3 normally inhibits the generation of soluble TNF by TACE, and that the increase in soluble TNF found in Timp $3^{-1-}$ mice leads to inflammation of the liver. The observed increases in the amounts of mature TACE and cell-associated TNF, as well as processed TNF, require further explanation. Soluble TNF might increase the expression of these proteins, either directly or as a result of the inflammation it induces (blue lines). Alternatively, TIMP3 might normally inhibit their expression (pink lines). The effects of TIMP3 on cell migration, angiogenesis and apoptosis (green bubbles) have been observed in tissue culture and upon overexpression in vivo. 
and treatment with a neutralizing antibody against TNF prevented the tissue damage.

Two caveats should be noted. First, a developmental defect might make Timp $3^{-/-}$mice more prone to produce TNF, although the only aberration in development that has been observed is in the lung ${ }^{14}$. A conditional knockout will be required to rule out a developmental cause of the phenotype. Second, the evidence for elevated TACE activity, increased cleavage of a peptide substrate by liver homogenates from Timp $3^{-1-}$ mice, is not conclusive. Like all known peptide substrates of TACE, the one used by the authors can be cleaved by other proteases. The authors show convincingly that the increase in cleavage (i.e., beyond that observed with liver homogenates from wild-type mice) is blocked by an inhibitor of TACE (TIMP3, in fact) and is not due to other reported TNF-processing enzymes. Nonetheless, in the absence of specific TACE inhibitors and substrates, interpretation of these results requires caution.

The greater peptide cleavage by liver extracts from Timp $3^{-1-}$ mice also leads to an interesting question. If the increased cleavage is due to increased TACE activity, is this elevated activity due to relief from TIMP3 inhibition or to the presence of a greater amount of TACE protein? This question arises because the authors note that there is an increase in the amount of mature TACE (the enzyme itself requires processing for activation) in liver extracts of Timp $3^{-1-}$ mice. Another twist is that in the absence of TIMP3, there is not only more processed TNF but also more of the cell-associated form of the cytokine. One explanation for this is that, after an initial increase in TNF processing due to relief from TIMP3 inhibition of TACE, the resultant increase in soluble cytokine induces (directly or indirectly) the expression of TNF itself and the processing enzyme (Fig. 1). A more radical hypothesis is that TIMP3 normally downregulates TNF and TACE expression, possibly through a cell-surface receptor or by blocking release of an inducer (Fig. 1).

\section{Insights into inflammation}

Whatever the relevant target, how TIMP3 reaches it is not clear, because TIMP3 binds to extracellular matrix components ${ }^{1}$. It is also unclear why spontaneous inflammation in Timp $3^{-1-}$ mice occurs only in the liver and not in the lung, gut or skin-other sites where the mice may be exposed to inflammatory stimuli from the environment. Both of the TNF receptors (I and II) are also TACE substrates, and perhaps in some tissues the increased release of TNF is balanced by an increase in soluble receptors.

If we set aside the two caveats noted above, and accept the less radical explanation for the increase in mature TACE protein and total TNF production (Fig. 1), this work settles two lingering issues in the TNF field: is the generation of soluble TNF important, and is TACE the main physiological TNF-processing enzyme? The answer seems to be yes, in both cases.

More broadly, whatever the mechanism, this paper suggests that TIMP3 has a physiological role in regulating TNF and inflammation. This conclusion has implications for understanding and treating human diseases.
For example, as the authors note, chronic hepatitis in humans involves lymphocytic infiltration as seen in Timp $3^{-1-}$ mice. An examination of TIMP3 levels, TACE activity and TNF production in individuals with hepatitis could therefore lead to a better understanding of this disease, and these parameters should be considered in the study of other inflammatory conditions as well. Moreover, if a deficiency of TIMP3 is detrimental, could elevating its levels be beneficial in inflammatory conditions? Adenoviral delivery to rheumatoid synoviocytes has already been tested ${ }^{4}$, and at least one antiarthritic agent increases TIMP3 expres$\operatorname{sion}^{15}$. The work by Mohammed et al. ${ }^{5}$ should stimulate many more such investigations.

1. Staskus, P.W., Masiarz, F.R., Pallanck, L.J. \& Hawkes, S.P. J. Biol. Chem. 266, 449-454 (1991).

2. Baker, A.H., Zaltsman, A.B., George, S.J. \& Newby, A.C. J. Clin. Invest. 101, 1478-1487 (1998).

3. Qi, J.H. et al. Nat. Med. 9, 407-415 (2003).

4. Van der Laan, W.H. et al. Gene Therapy 10, 234-242 (2003).

5. Mohammed, F.F. et al. Nat. Genet. 36, 969-977 (2004)

6. Lambert, E., Dasse, E., Haye, B. \& Petitfrere, E. Crit. Rev. Oncol. Hematol. 49, 187-198 (2004).

7. Baker, A.H., Edwards, D.R. \& Murphy, G. J. Cell Sci. 115, 3719-3727 (2002).

8. Nagase, H. \& Woessner, J.F. Jr. J. Biol. Chem. 274 21491-21494 (1999).

9. Black, R.A. Int. J. Biochem. Cell Biol. 34, 1-5 (2002).

10. Moss, M.L. et al. Nature 385, 733-736 (1997).

11. Amour, A. et al. FEBS Lett. 435, 39-44 (1998).

12. Lee, M.-H. Biochem. J. 364, 227-234 (2002).

13. Wielockx, B. et al. Nat. Med. 7, 1202-1208 (2001).

14. Leco, K.J. et al. J. Clin. Invest. 108, 817-829 (2001).

15. Takizawa, M. et al. Arthritis Rheum. 43, 812-820 (2000).

\title{
The G-netics of dark skin
}

\author{
Ian J Jackson
}

Several mutant strains of mice have dark skin pigmentation due to an aberrant accumulation of pigment-producing melanocytes in the dermal layer of the skin. A new study shows that three such strains carry activating mutations in the genes encoding the Gprotein subunits $\mathrm{G} \alpha q$ or $\mathrm{G} \alpha 11$, resulting in more pigment cell precursors and an excess of dermally retained pigment cells at birth.

Mutations that affect mouse pigmentation have been studied for over a century. But despite this long history, large-scale mutagenesis programs ${ }^{1,2}$ continue to identify new genes involved in this process. A series of ten dominant dark skin (Dsk) mutations recently described by Greg Barsh and colleagues ${ }^{3}$ can

Ian J. Jackson is at the MRC Human Genetics Unit, Western General Hospital, Edinburgh, UK.e-mail:ian.jackson@hgu.mrc.ac.uk be classified into two categories, depending on whether dermal or epidermal pigmentation is increased. On page 961 of this issue, Van Raamsdonk et al. ${ }^{4}$ report that three of the four Dsk mutants with increased dermal pigmentation carry activating mutations in Gnaq and Gna11. These genes encode functionally related $\mathrm{G} \alpha$ subunits that mediate the first step in transducing signals from $G$ protein-coupled receptors, identifying a key role for these genes in regulating skin color.

\section{The road less traveled}

Melanoblasts, the precursors of the pigmentproducing cells of the skin and hair, arise during development from a migratory cell population called the neural crest. Some of the key molecules required in the developmental path from neural crest to melanoblast include the transcription factors Pax3, Sox10 and Mitf and the cell-surface receptors Kit and Ednrb ${ }^{5}$. As the two layers of the skin develop, melanoblasts enter the dermis and then 